\section{Unspoken fears}

SIR - With regard to safety provisions for environmental releases, organisms genetically modified by recombinant DNA techniques are often singled out as intrinsically risk-entailing. This widespread attitude leads to scientifically untenable distinctions, such as the inclusion of site-directed mutagenesis among the techniques to be kept under scrutiny, and the simultaneous exemption of random mutagenesis. By equating 'natural' with 'safe', this prejudice grossly underestimates the risks posed by incautious environmental introductions of 'natural' organisms. The most dramatic predictions made for recombinant organisms apply equally to conventional ones.

Excessive proliferation of conventional organisms in the wake of their "planned' introduction (rabbits in Australia, the Nile perch in Lake Victoria) has already caused ecological disasters. Mechanisms allowing horizontal gene transfer do not distinguish between recombinant and conventional organisms, and the spread of a natural, nonrecombinant plasmid conferring resistance to an antibiotic in use represents a real danger, which should be rigorously kept in check. New, undesirable characters can emerge unpredictably as a result of conventional breeding ${ }^{1}$.

Such considerations reinforce the concept that, within the spectrum of practicable genetic modification techniques, the more precise the method of introducing a new trait, the lower the likelihood of an unpredicted effect. What generates confusion is the lack of accepted, general classes of environmental risk, similar to those applied in issues relating to human health. Leaving environmental risks in the realm of imagination continues to fuel the idea that environmental introductions of recombinant organisms entail unique risks.

I have drawn up a tentative classification of possible environmental risks, ranked according to level of concern (see table). The assignment of an organism,

\section{PROPOSED CLASSIFICATION OF ENVIRONMENTAL} RISK GROUPS

Organisms which were never recognized to pose a risk to the environment

Organisms which may cause transient ecological imbalances or transient biogeochemical effects

Organisms which might be pathogens or pests for plants or animals, but have limited diffusion or persistence

Organisms which might transfer unwanted genetic traits to other species

- Organisms which may cause persistent undesirable ecological imbalances or persistent biogeochemical changes

Dangerous and highly diffusive pathogens or pests, either for animals or for plants

whether natural or modified, to one of the risk classes would be based on the properties of the organism and of the target environment. A careful description of the parental organism, of the traits added (or deleted) and of the nature and precision of the modification would appear among the attributes to be considered in assessing genetically modified organisms ${ }^{2}$.

Such a tentative list of possible environmental risks, which might easily be extended to include effects specifically attributable to recombinant DNA organisms, were any to be identified, was brought to the attention of the OECD group of national experts on safety in biotechnology at its plenary meeting in Paris on 26 June 1991. My approach was rejected, being labelled "premature".

May I ask whether it is still "premature", after so many years of debate, to try to state what are the real concerns? Or is a pyramid of power and regulation being built on unspoken fears?

ClaRA Frontal

Laboratorio de Biologia Cellulare,

Istituto Superiore de Sanità.

Viale Regina Elena 299,

O0161 Rome, Italy

1. Day, P.R. Swiss Biotech 5, 39 (1987)

2. Miller, H.I., Burris, R.H., Vidaver, A.K. \& Wivel, N.A. Science 250, 490 (1990)

\section{Milky way}

SIR - I have recently been in Nigeria, where an undereducated population is constantly exposed to advertising campaigns, notably by Nestlé, in which the full strength of current methods of persuasion is used to convey the seductive impression that formula feeding is the modern, westernized thing to do and will result in a bouncing healthy baby (and a happy smiling husband, too!). When the truth of these campaigns is apparently confirmed by gifts of free samples of formula from trusted clinics, mothers can hardly be said to be making a 'free choice' (Nature 352, 266; 1991).

44 Porchester Square, London W2 6AW

\section{Climate change}

SIR - The contention of Jesse H. Ausubel (Nature 350, 649; 1991) that the world need not worry unduly about climate change because it has developed or can develop the technology for adaptation sounds rather hollow in developing countries. Those who have the money and the technology may be able to protect themselves against changes in climate. But has Ausubel given any thought to the vast majority of people on the globe who live in poverty? How would they be able to cope with the vagaries of climate change, which in the first place would be overwhelmingly a consequence of the technological 'advancement' of the rich nations? Even the rich countries may not be fully able to protect themselves against a very drastic change in climate.

Witness the recent havoc caused by a cyclonic storm in Bangladesh, where up to 300,000 people may have died, in spite of advance warning. Since then, Bangladesh has been battered by a series of storms in which several thousands have died. Here we are talking about 'normal weather' and not climate change as yet. What would happen if, as predicted by many models, there is an increase in freak storms? How would Bangladesh raise the resources to protect its vulnerable coastline and its people? How would the Maldives, a tiny nation of islands in the Indian Ocean, or other developing countries cope with rising sea levels caused by a warmer Earth? For that matter, can even a moderately rich nation such as the Netherlands save its precious land from the onslaught of the rising oceans if the increase in mean sea level is very high?

R. SUKUMAR

K. V. DEVI PRASAD

Centre for Ecological Sciences,

Indian Institute of Science, Bangalore 569 012,

India

\section{Watching the ball}

SIR - With a background in physics as well as molecular biology, I believe I am a "qualified scientist" who has indeed observed ball lightning (Nature 350, 108; 1991). The following is a report I made immediately afterwards.

"During an incredible electrical storm [in Houston, Texas on 18 June 1991] in the evening while sitting at a table in the breakfast room, I saw a ball of lightning enter the utility room [an extension of the breakfast room] apparently through the back door. It hovered as a revolving sphere of bright yellow, orange and red light about 10 inches in diameter, in the air about three feet above the floor. It stayed in the same place. After about two or three seconds the globe disappeared with a loud pop rather like a discharge from a champagne bottle. This discharge was followed by a distinct odor of ozone. My Siamese cat also appeared to see the ball; at least he ran towards it."

This experience was amazing and interesting without being in any way frightening. My main thought was how much we scientists still do not know about natural phenomena.

\section{Department of Microbiology} and Immunology,

Baylor College of Medicine, One Baylor Plaza,

Texas Medical Center,

Houston, Texas 77030 , USA 